\section{La vision japonaise de la géographie humaine}

\section{Jean-Robert Pitte}

(Université de Paris-Sorbonne)

The Human Geography est une revue généraliste japonaise de grande qualité. Elle est rédigée en japonais, mais chaque article est accompagné de substantiels résumés en anglais. Il est simplement dommage que les légendes cartes et des graphiques ne soient pas également traduites, de même que les titres des ouvrages dont le compte-rendu est donné en fin de livraison. Ceci donnerait à la revue une plus grande audience internationale et permettrait à un certain nombre d'illustrations d'être réutilisées dans les manuels de géographie paraissant dans les autres grandes langues scientifiques.

Les auteurs de la revue sont à la fois des seniors et des juniors de la discipline, ce qui représente un excellent moyen de souder une communauté scientifique et de préparer le renouvellement des thématiques. La variété des sujets traités révèle une véritable liberté de ton et une absence de dogmatisme. Cette revue n'est pas celle d'une école, mais de plusieurs tendances. Elle publie des articles théoriques et conceptuels, mais aussi des monographies, assez nombreuses abordant tous les aspects de la géographie humaine. Il faut saluer la place de choix accordée à la géographie historique. Cette approche pluridisciplinaire a connu des heures de gloire en France jusque dans les années 1960. Les historiens et les géographes ont ensuite divergé, mais depuis une dizaine d'années, de nouvelles collaborations s'instaurent. Une commission de géographie historique vient d'être recréée au sein du Comité National Français de Géographie. Il faut s'en réjouir et abandonner le réflexe de repli communautaire qui enferme la géographie au lieu de la rendre nécessaire à toutes les autres sciences de l'Homme et de son environnement.
Sur ce dernier thème, précisément, The Human Geography a publié une remarquable contribution en anglais de J. M. Powell (1999, 5). L'une des spécificités de la géographie consiste à étudier la relation objective et subjective entre l'humanité et son environnement. C'est une des branches essentielles de la géographie. Elle permet de dépasser l'archaïque clivage géographie physique - géographie humaine. Il faut néanmoins signaler l'article de Shizuyo Sano $(1999,4)$ qui aborde à la fois les questions d'environnement, d'agriculture irriguée, de géographie politique et sociale en montrant le rôle des seigneurs au Moyen Age dans la maîtrise du milieu japonais.

Plusieurs textes parus dans les années 19992000 appellent quelques commentaires libres. Les voici très simplement, pour ouvrir la discussion.

- Yoshio Sugiura évoque les travaux d'Edward Ullman (1999, 1). L'analyse spatiale est-elle une manière si neuve de concevoir et pratiquer la géographie. N'y a-t-il pas de la part de certains chercheurs un peu $\mathrm{d}^{\prime}$ arrogance intellectuelle à vouloir remplacer géographie par «analyse spatiale». Le quantitatif et le qualitatif ne s'excluent nullement. Ce n'est pas admis par tous les chercheurs, mais certaines réalités ne peuvent s'appréhender que qualitativement. Celles que des sources statistiques fiables permettent d'approcher quantitativement demandent toujours pour être interprétées une analyse fine qui marie l'usage des modèles éprouvés et le jugement intuitif faisant appel à différents facteurs explicatifs. La frontière entre science dure et science molle s'estompe chaque jour un peu plus.

- L'échec relatif de la production forcée des mandarines, étudié par Atsushi Kawakubo $(1999,2)$ est assez révélateur de la crise grave que traverse l'agriculture productiviste dans le monde. Considérée pendant longtemps comme une panacée, elle montre aujourd'hui 
clairement ses limites. Elle repose sur un calcul économique absurde, tant les intrants sont élevés, tout comme, par conséquent, le prix de vente de ses produits, inaccessibles aux consommateurs des pays pauvres qui ne peuvent donc absorber les excédents des pays riches. En outre, elle coûte cher aux contribuables, puisqu'elle vit de subventions directes ou indirectes. Elle pollue l'environnement, tant elle utilise d'engrais et de pesticides. Elle homogénéise les paysages et prive ceux qui y vivent ou qui les fréquentent en touristes d'émotions intenses. Elle déverse sur le marché des produits insipides, eux aussi dépourvus de toute faculté d'émouvoir. Il est temps de revenir à une agriculture plus proche de la réalité géographique.

- Kazuaki Sugiyama $(1999,4)$ étudie en détail l'espace social d'un quartier de nuit (Ekimae à Toyama). La géographie sera aimée des non géographes si elle s'intéresse à l'espace vécu des sociétés et apporte quelques clés de compréhension pour celles-ci, quelques moyens d'améliorer leur bien-être.

- À la fois de géographie historique et politique, l'article de Kunitada Narumi $(1999,6)$ démontre les qualités de cartographes des villageois japonais au début de l'époque d'Edo. Il aborde la question fondamentale de la connaissance de l'espace par les sociétés préindustrielles. Pour elles les superficies et les altitudes étaient aussi importantes que pour les sociétés industrialisées. Dans un contexte de forte pression fonciére et de bon niveau d'éducation - l'un des plus hauts du monde pour une société rurale à cette époque-une représentation aussi fidèle que possible de l'espace était vitale.

- L'article de Neil Smith (2000, 1 ; en anglais) aborde la question centrale de la mondialisation. Lorsqu'on est à la fois géographe et historien, on se rend compte que cette inéluctable réalité n'est ni bonne, ni mauvaise. Tout dépend de la manière dont on l'utilise. Il est certain que les pays pauvres sont actuellement fragilisés par rapport à l'accélération des échanges qu'elle provoque. Des solutions raisonnables et équitables sont possibles. Elles demandent sens des responsabilités, imagination et génerosité.

- Koji Ohnishi $(2000,2)$ traite d'un sujet original : l'espace perçu et vécu par les enfants. Il rattache son étude à la géographie des genres, très en vogue dans le monde anglo-saxon et très peu en Europe latine. Il suggère l'intérêt d'aborder cette question du point de vue socio-économique plutôt qu'individuel. Pourtant, les deux approches ne sont pas contradictoires, car l'Homme est un animal social, mais il a aussi une personnalité individuelle et l'organisation de l'espace dépend de ces deux facettes qu'il n'est pas raisonnable d'opposer.

- Honami Kageyama $(2000,4)$ s'intéresse aux appartements attribués à Otsuka aux femmes salariées dans les années 1930. C'est une originale géographie des genres. L'étude des manières d'organiser l'espace $\mathrm{d}^{\prime}$ habitation est en plein renouveau. Elle fait enter dans le champ de la géographie l'espace intérieur, alors que seul l'extérieur (baptisé «paysage») faisait jusqu'à ces dernières années l'objet des études les plus nombreuses.

- L'organisation des brasseurs de saké pendant l'ère Meiji peut sembler un sujet éloigné de la géographie. Pourtant, Takahiro Aoki (2000, 5 ) en tire de très utiles enseignements. Il n'existe pas de petit sujet : tout dépend de la manière dont on le traite. En l'occurrence la description de l'amélioration de la qualité, comme moyen de dépasser la concurrence entre fabricants est une leçon très utile dans la situation agricole du début du XXIe siècle. Ceci rejoint le problème de la crise des cultures intensives de mandarines.

- Les questions touchant à l'industrie et aux services les plus modernes ne sont guère 
abordés dans The Human Geography qui accorde beaucoup d'importance au passé. L'article de Hidekazu Aoki $(2000,5)$ fait exception. Il décrit les principes de localisation des établissements Sony et l'impact des usines sur les régions choisies. Il est essentiel de renouveler l'étude des facteurs de localisation des industries et des services en soulignant tout ce qui relève de la culture régionale et d'entreprise. La géographie économique traditionnelle, plutôt tournée vers les coûts apparents ne s'y était guère intéressée. Saluons l'évolution! 